\title{
Criminologie
}

\section{Comparaison franco-britannique de la recherche sur les services de renseignement}

\section{Olivier Chopin et Bastien Irondelle}

Volume 46, numéro 2, automne 2013

URI : https://id.erudit.org/iderudit/1020985ar

DOI : https://doi.org/10.7202/1020985ar

Aller au sommaire du numéro

\section{Éditeur(s)}

Les Presses de l’Université de Montréal

ISSN

0316-0041 (imprimé)

1492-1367 (numérique)

Découvrir la revue

Citer cet article

Chopin, O. \& Irondelle, B. (2013). Comparaison franco-britannique de la recherche sur les services de renseignement. Criminologie, 46(2), 15-42. https://doi.org/10.7202/1020985ar
Résumé de l'article

Alors que la publication devenue " industrielle " de recueils et de manuels sur les études du renseignement (Intelligence Studies) démontre l'arrivée à maturité des études sur le renseignement au sein de la science politique internationale, la recherche universitaire sur le sujet demeure en France au stade de l'émergence. Le retard français par rapport au Royaume-Uni se double d'un second sur le décalage entre faible connaissance et importance des services de renseignement, la France étant un des rares pays à disposer d'une panoplie complète de services de renseignement intérieurs et extérieurs, civils et militaires, et de moyens techniques de premier plan. L'article présente l'état actuel des études sur le renseignement en France et au Royaume-Uni, analyse la trajectoire du développement de ce champ de recherches et avance des hypothèses sur la réussite britannique et le développement tardif et fragile des études sur le renseignement français. L'explication tient au fonctionnement du marché universitaire, aux transformations de l'État et des modalités de la reconnaissance institutionnelle et sociale du renseignement, et enfin à la mécanique d'un processus différencié de relégitimation du renseignement sur les deux rives de la Manche. 


\title{
Comparaison franco-britannique de la recherche sur les services de renseignement
}

\author{
Olivier Chopin ${ }^{1}$ \\ Enseignant \\ Sciences Po (Paris) \\ Chercheur associé \\ École des hautes études en sciences sociales (EHESS) \\ olivier.chopin@ehess.fr \\ Bastien Irondelle \\ Professeur associé \\ Sciences Po (Paris) \\ Chercheur \\ Centre d'études et de recherches internationales (CERI) \\ bastien.irondelle@sciences-po.fr
}

RÉSUMÉ - Alors que la publication devenue «industrielle» de recueils et de manuels sur les études du renseignement (Intelligence Studies) démontre l'arrivée à maturité des études sur le renseignement au sein de la science politique internationale, la recherche universitaire sur le sujet demeure en France au stade de l'émergence. Le retard français par rapport au Royaume-Uni se double d'un second sur le décalage entre faible connaissance et importance des services de renseignement, la France étant un des rares pays à disposer d'une panoplie complète de services de renseignement intérieurs et extérieurs, civils et militaires, et de moyens techniques de premier plan. L'article présente l'état actuel des études sur le renseignement en France et au Royaume-Uni, analyse la trajectoire du développement de ce champ de recherches et avance des hypothèses sur la réussite britannique et le développement tardif et fragile des études sur le renseignement français. L'explication tient au fonctionnement du marché universitaire, aux transformations de l'État et des modalités de la reconnaissance institutionnelle et sociale du renseignement, et enfin à la mécanique d'un processus différencié de relégitimation du renseignement sur les deux rives de la Manche.

MOTS-CLÉS - Renseignement, études sur le renseignement, services secrets, France, Grande-Bretagne.

1. Olivier Chopin, École des Hautes Études en Sciences Sociales, CESPRA, 105 boulevard Raspail, 75018, Paris, FRANCE 


\section{Introduction}

Alors que la publication devenue «industrielle» de recueils et de manuels sur les études du renseignement (Intelligence Studies) (Johnson, 2006, 2010; Jackson, sous presse) démontre l'arrivée à maturité, parfois jusqu'à la redondance, d'un champ intellectuel des études sur le renseignement, en particulier aux États-Unis, Canada et Royaume-Uni, la recherche universitaire sur le renseignement demeure en France au stade de l'émergence, si l'on reprend les étapes de l'historiographie des études $\mathrm{du}$ renseignement britannique proposées par Moran (2011). Cette phase, marquée par le rôle décisif des historiens, a laissé place au Royaume-Uni à la phase actuelle d'efflorescence des études sur le renseignement. Ce premier constat d'un retard français par rapport au Royaume-Uni se double d'un second sur le décalage entre l'importance des services de renseignement français (la France étant l'un des rares pays à disposer de l'ensemble des services de renseignement intérieurs et extérieurs, civils et militaires et possédant des moyens techniques de premier plan en matière de renseignement électronique - plus communément désigné Signal Intelligence, ou SIGINT -), la place qu'ils occupent dans l'État et ses politiques régaliennes, dans la politique étrangère et la puissance militaire, et le caractère encore embryonnaire de l'étude universitaire de ces sujets: aucune maîtrise (master) spécialisée, une poignée d'enseignants-chercheurs, aucun auteur français dans les différents recueils cités. L'objectif de cet article est double. Il s'agit, d'une part, de présenter l'état actuel des études sur le renseignement en France et au Royaume-Uni, d'analyser la trajectoire du développement de ce champ de recherches. Il s'agit, d'autre part, d'avancer des hypothèses expliquant la réussite britannique et le développement tardif et fragile en France des études sur le renseignement. Il convient de préciser que le périmètre consenti à cet article nous oblige à nous limiter à la recherche strictement universitaire dans une définition stricte des études du renseignement, en assumant d'exclure «l'intelligence économique» (Dénécé \& Arboit, 2010) et les études fortement techniques sur la cryptographie (Kahn, 2008), bien étudiées par ailleurs (Kahn, 2008; Dénécé \& Arboit, 2010). La première partie de l'article explore le «printemps français» des études sur le renseignement, la seconde partie analyse le développement spectaculaire des Intelligence Studies au Royaume-Uni. La dernière partie étaye quelques hypothèses pour expliquer ce décalage constaté entre la France et le Royaume-Uni en matière d'études sur le renseignement. 


\section{France : un «printemps » des études sur le renseignement?}

Avant de le comparer avec la situation britannique, il est intéressant de relater rapidement l'état actuel des études sur le renseignement sur le plan national. Plusieurs initiatives constituent l'embryon de recherches universitaires sur lesquelles un «jeu d'hypothèses» national peut désormais être proposé, qui représente peut-être un «printemps des études françaises sur le renseignement».

\section{La gestation du champ universitaire sur le renseignement}

Au cours de la dernière décennie, les études sur le renseignement ont connu un essor important à partir des travaux fondateurs de l'amiral Pierre Lacoste. L'un des points d'émergence des études historiques du renseignement peut être placé dans la première publication du Centre d'études d'histoire de la défense (CEHD), créé en 1994. Le Cahier n 1 daté de 1996 s'intitule Histoire du renseignement et publie six premiers travaux issus de conférences menées dans le cadre de la Commission d'histoire du renseignement, l'une des toutes premières instances académiques à traiter du renseignement en France, sous l'impulsion de Bertrand Warusfel $(1998,2007)$.

L'amiral Pierre Lacoste, ancien directeur de la Direction générale de la Sécurité extérieure (DGSE, l'équivalent de la CIA américaine ou du Service canadien du renseignement de sécurité), a tenu un rôle à la fois fondateur et fédérateur dans l'émergence des études françaises sur le renseignement. En effet, il est le fondateur d'une «posture» et d'un «idéal» de constitution d'un mouvement de recherche transdisciplinaire. C'est lui qui pose l'enjeu d'aboutir un jour à une authentique école française du renseignement. Il fixe le consensus national sur la situation des études en France: celles-ci sont interprétées en termes de «culture du renseignement». Cette formule désigne par la suite en même temps la configuration des études en France, le diagnostic d'un déficit, voire d'un problème structurel, et la variable explicative de cette situation (Lacoste, 1997, 1998).

De 2006 à 2010, un programme de l'Agence nationale de la recherche a été spécifiquement dédié au renseignement, il s'agit du programme «Information ouverte information fermée» (IOIF) dirigé par Sébastien Laurent. Le projet scientifique originel de l'IOIF repose 
sur «le constat d'une absence, en France, de travaux historiques sur ce thème alors que les Intelligence Studies connaissaient en Grande-Bretagne et en Amérique du Nord un fort développement depuis une vingtaine d'années ${ }^{2} »$. L'ambition de Sébastien Laurent était de réunir des chercheurs avec le plus large horizon disciplinaire possible. Il s'agit d'une réalisation qui fait émerger, dans une perspective historienne, une authentique recherche universitaire de long terme et structurée. Trois ouvrages majeurs ont été publiés dans le cadre de cette ANR (Laurent, 2009a, 2009b, 2011a). En outre, ce programme IOIF s'articule avec les recherches personnelles de Sébastien Laurent: ses enseignements communs avec Olivier Forcade ont donné lieu à une publication dès 2005 (Forcade \& Laurent, 2005). En parallèle, Laurent a travaillé, à partir d'une vaste réflexion épistémologique archivistique, sur les problématiques de l'accès aux sources primaires et à la classification (Laurent, 2003). Il a enfin récemment publié sa thèse d'Habilitation à diriger des recherches (HDR) (Laurent, 2009c) qui porte principalement sur le renseignement $\mathrm{du} \mathrm{XIX}^{\mathrm{e}}$ siècle jusqu'à la Première Guerre mondiale.

Depuis le début de l'année 2008, Sébastien Laurent anime avec Olivier Forcade et Philippe Hayez $z^{3}$ le groupe METIS au sein du Centre d'histoire de Sciences Po à Paris. Son principe consiste à proposer deux cycles de conférences-discussions, appelées «saisons», par année universitaire, une plateforme pour les études sur le renseignement, qui regroupe chercheurs, anciens des services et étudiants.

Sous l'impulsion des recherches d'Olivier Forcade, l'Université Paris IV-Sorbonne a élargi les classiques approches historiques et militaires du renseignement à l'approche plus internationaliste. Olivier Forcade a soutenu une HDR en décembre 2005 à l'Université Paris IV (Forcade, 2005), cette étude a été publiée trois ans plus tard (Forcade, 2008). Louvrage est une exploration méthodique des archives des services spéciaux militaires et, à un moindre degré, de celles de la sûreté générale. En analysant le renseignement essentiellement par le biais d'une approche des relations internationales, de l'histoire stratégique et militaire, ou encore de l'analyse des politiques du renseignement dans les États (Forcade, 2001, 2005), l'Université Paris IV doit être consi-

2. Source: Agence Nationale de la Recherche, ANR. Consulté le 6 juillet 2013, www. agence-nationale-recherche.fr/projet-anr/? tx_lwmsuivibilan_pi2\% 5BCODE \% 5D=ANR-06-JCJC-0153

3. Philippe Hayez est conseiller-maître à la Cour des comptes, ancien directeur adjoint de la Direction du renseignement de la DGSE. 
dérée comme un autre lieu privilégié du développement des études de renseignement dans les sciences humaines et sociales en France.

Pour compléter cette brève recension, il est important de souligner en outre la vitalité du récent filon des publications en histoire du renseignement. Parmi les travaux les plus récents, on peut citer ceux de Laurent (2011b), de Forcade et al. (2010), de Faligot et al. (2012). Si, comme le remarque Gérald Arboit (2013) dans un récent rapport de recherche, le renseignement peut encore apparaître comme une «dimension manquante de l'histoire contemporaine de la France», celle-ci tend malgré tout à se réduire à mesure que les travaux historiques progressent et s'accumulent. En outre, il convient de remarquer que les historiens français s'inscrivent nettement dans la perspective de l'histoire immédiate (ou «histoire du temps présent»). Du côté de la science politique, en revanche, la situation paraît beaucoup moins florissante, bien que plusieurs recherches doctorales importantes aient été menées à bien ces dernières années: l'une sur l'anti-terrorisme aux États-Unis (Bonditti, 2007); une autre sur la coopération anti-terroriste en Europe (Guittet, 2006), une troisième sur le rôle de l'exécutif dans la lutte anti-terroriste en France (Vadillo, 2012), une quatrième sur le contrôle parlementaire des services de renseignement (Lepri, sous presse), une dernière sur les services de renseignement au prisme de la raison d'État (Chopin, 2005).

\section{L'offre de formation universitaire}

L'offre de formation demeure rare en France sur les questions de renseignement, si on la compare à celle du Royaume-Uni ou du Canada. Notamment, il n'existe aucune maîtrise spécialisée. À l'Université Paris IV-Sorbonne, un séminaire de recherche de $2^{\mathrm{e}}$ année de maîtrise (Master 2 ) et doctoral sur «le renseignement dans les relations internationales aux XX-XXI ${ }^{e}$ siècles» a été ouvert en 2008 par Olivier Forcade, parallèlement au séminaire METIS de Sciences Po évoqué supra. Ce séminaire de recherche sur l'histoire du renseignement est annuel, avec deux heures hebdomadaires sur 25 semaines, et présentant une approche d'histoire des relations internationales. Le thème de l'année 2008-2009 était dédié à «la décision, le renseignement dans les relations internationales de 1914 à 1989 », celui de 2009-2010 portait sur «les coopérations internationales de renseignement dans les relations internationales de 1914 à 2001 ». Le programme de 2010-2011 fut consacré aux «communautés nationales de renseignement dans les 
relations internationales de 1940 à $2010 »$, afin d'interroger la notion proposée. On peut également citer le Master 2 professionnel intitulé «Sécurité globale et analyste trilingue» à l'Université Bordeaux IV qui accorde depuis deux ans une part importante sur deux semestres aux questions de renseignement.

Sciences Po Paris propose depuis plusieurs années des cours sur le renseignement. On remarque que l'offre d'enseignement s'est notamment concentrée au sein de la maitrise «International Security» de la Paris School of International Affairs, qui propose désormais une concentration thématique sur le renseignement. Animée par Philippe Hayez, cette spécialisation s'articule autour de deux cours magistraux au premier semestre et de séminaires spécialisés au second semestre. Sciences Po représente sans doute à ce jour l'offre la plus importante d'enseignements sur le renseignement dans le cadre d'une formation généraliste de l'enseignement supérieur.

Le caractère encore embryonnaire des recherches menées en France s'accompagne d'un aspect lacunaire des travaux et des résultats du côté de la science politique et surtout des études sur la sécurité (security studies). Le contraste est d'autant plus saisissant que ce champ universitaire naissant fait face à une tradition universitaire beaucoup plus ancienne et fournie dans le monde anglo-saxon, avec un volume de travaux important, des revues scientifiques renommées, des équipes de recherche internationales situées dans les universités les plus prestigieuses. Le cas britannique détaillé ci-dessous permet de prendre la mesure de ce contraste spectaculaire.

\section{Royaume-Uni : le développement spectaculaire des études sur le renseignement}

Comme dans le cas des États-Unis, le milieu des années 1970 constitue une étape charnière dans le développement des études sur le renseignement au Royaume-Uni (Goodman, 2006). Jusqu'à ce moment, la plupart des ouvrages abordant le renseignement et l'espionnage sont des mémoires, des biographies ou des récits d'opérations spécifiques. La publication de deux ouvrages universitaires sur Ultra, le programme britannique de décodage des communications allemandes pendant la Seconde Guerre mondiale, marque l'entrée du renseignement dans le champ de la recherche universitaire (Masterman, 1972; Winterbotham, 1974). Les études sur le renseignement vont alors connaître durant les 
trois décennies suivantes un développement spectaculaire, dominé au départ par l'histoire, avec la figure tutélaire de Christopher Andrew. Par la suite, elles vont se diversifier vers la science politique et les relations internationales. On peut dégager deux grandes périodes dans les études britanniques sur le renseignement. La première phase de développement et de consolidation se traduit notamment par l'ouverture de nouveaux champs disciplinaires (science politique, relations internationales, criminologie) après une période initiale où seule l'histoire s'intéressait au renseignement. Elle couvre la période qui court de la fin des années 1970 au début des années 1990. La seconde phase concerne les deux dernières décennies: elle est marquée par l'accélération et l'approfondissement des études sur le renseignement. À partir du milieu des années 1980, ces études vont être stimulées à la fois par l'extraordinaire développement des intelligence studies aux États-Unis et par les premiers enseignements sur le renseignement offerts dans les universités britanniques. Par ailleurs, la création en 1986 d'une revue de référence, Intelligence and National Security, par Christopher Andrew et Michael Handel joue un rôle de catalyseur dans le développement des études sur le renseignement et favorise l'essor de la pluridisciplinarité. La revue est aujourd'hui codirigée par Loch Johnson et Peter Jackson.

On peut, à certains égards, parler d'un «boom», encouragé et facilité par les scandales liés à la polémique concernant les armes de destruction massive en Irak lors de la guerre de 2003. La publication de nombreux rapports officiels va ainsi donner accès à un matériau primaire, jusquelà peu disponible. Dans le même moment se développent les histoires officielles des services de renseignement qui fleurissent à la fin des années 2000.

\section{Le dynamisme de l'enseignement sur le renseignement au Royaume-Uni}

L'université britannique compte au moins trois centres de recherche spécialisés sur les questions de renseignement. Le Centre for Intelligence and International Security Studies de l'Université d'Aberystwyth; le Brunel Centre for Intelligence and Security Studies, créé sous l'impulsion de Philippe Davies; le Buckingham University Center for Security and Intelligence Studies autour de Anthony Glees.

Il faut cependant relever que conformément à la pratique des universités au Royaume-Uni, ces centres de recherche sont souvent de taille 
relativement restreinte et animés par deux ou trois titulaires et un certain nombre de chercheurs associés et de doctorants. Dans d'autres cas, le centre de recherche n'est pas entièrement ou principalement dédié aux études sur le renseignement, mais celles-ci constituent une part significative de l'activité de recherche et de publication avec plusieurs chercheurs spécialisés. C'est notamment le cas du King's College de Londres où l'on trouve des spécialistes du renseignement (historiens et/ ou politistes) tant dans le Defence Studies Department que dans le War Studies Department (Lawrence Freedman, David Oman, Christina Goulter, Michael Goodman). Certaines universités, sans disposer de centres de recherche spécialisés, constituent cependant des pôles d'excellence et d'attraction. C'est évidemment le modèle de l'Université de Cambridge autour de l'Intelligence Seminar du département d'histoire animé par Christopher Andrew ou de l'Oxford Intelligence Group organisé au sein du Nuffield College à Oxford autour de Michael Hermann. Si on lui ajoute Richard Aldrich, l'une des figures de proue des études sur le renseignement à l'Université de Warwick, Robert Dover à la Loughborough University ou Mark Phythian à Leicester, plus quelques chercheurs sur le sujet «isolés» dans certains départements, on parvient à la conclusion que quasiment chaque université de premier plan et chaque département important de relations internationales compte un spécialiste du renseignement dans son corps professoral.

Pas moins de six universités proposent une maîtrise spécialisée, qui est souvent fortement multidisciplinaire: University of Wales Aberystwyth, King's College London, University of Salford, University of Birmingham, Brunel University, Buckingham University. Le département de relations internationales de l'University of Wales, Aberystwyth, l'un des plus importants et des plus dynamiques, propose pas moins de quatre programmes de maîtrise: Master of Sciences en Intelligence and Strategic Studies; en Intelligence Studies and Internatinal History; en Intelligence Studies; et un Master of Arts en Intelligence.

Sans que les frontières soient totalement étanches, deux profils de formation se dégagent: l'une plutôt universitaire destinée à des étudiants s'intéressant au renseignement au sein d'un cursus plus large de science politique ou d'histoire, certains pouvant être intéressés par une carrière dans le renseignement; l'autre plus clairement orientée vers des professionnels du renseignement ou plus encore des professionnels qui doivent connaître le renseignement. L'une des conclusions du rapport Butler, Review of Intelligence on Weapons of Mass Destruction, concernait 
la faiblesse de l'analyse dans le cycle du renseignement. Le groupe chargé de la mise en pratique des recommandations du rapport Butler visait notamment à développer une formation plus substantielle que jusqu'à maintenant pour tous les analystes sur le plan interministériel, voire de nommer des universitaires au sein même de la structure d'analyse de haut niveau (Her Majesty's Stationery Office (HMSO), 2004). L'un des rôles du Professional Head of Intelligence Analysis (PHIA), fonction nouvellement créée, consiste à réduire le fossé entre le monde universitaire et Whitehall (Her Majesty's Stationery Office (HMSO), 2005; Goodman, 2006). King's College et Brunel University sont particulièrement à la pointe sur ces formations qui visent à familiariser un large éventail de hauts fonctionnaires venant de ministères a priori ou traditionnellement peu concernés par les problématiques du renseignement (éducation, immigration, santé...) (Goodman \& Omand, 2008).

\section{De nouveaux gisements de sources: «saison des enquêtes » et histoire officielle}

Dans le contexte actuel, après la guerre en Irak, les enquêtes officielles qui permettent la déclassification d'un nombre inhabituel de documents constituent une des sources principales d'information. En effet, les conditions de l'engagement militaire britannique et la question de l'utilisation, voire le soupçon de manipulation, du renseignement par le pouvoir politique d'une part, et le suicide de David Kelly, expert en armes de destruction massive du ministère de la Défense accusé d'être la source à l'origine des révélations de la $\mathrm{BBC}$ sur les pressions exercées par le cabinet du premier ministre sur les services de renseignement pour crédibiliser la menace irakienne d'autre part, ont conduit à quatre enquêtes parlementaires successives ou ad hoc qui ont produit des rapports substantiels sur les questions de renseignement. Il s'agit de celui de la Commission des affaires étrangères du Parlement ${ }^{4}$, des rapports de l'Intelligence $\mathfrak{\sigma}$ Security Committee, du rapport de la commission Hutton sur la mort de David Kelly de 2004 et du rapport de la commission Butler de 2004 sur les performances du renseignement britannique concernant les armes de destruction massive irakiennes 5 .

4. Source: Federation of American Scientists. Consulté le 6 juillet 2013, www.fas.org/ irp/threat/ukiraq0703.pdf

5. L'ensemble de ces documents est rassemblé sur le site de la Federation of American Scientists. Consulté le 6 juillet 2013, www.fas.org/irp/world/uk/index.html 
La pratique de l'histoire officielle comme moyen de développer la connaissance et la recherche universitaire sur le renseignement est également importante, mais n'est pas sans faire débats parmi les historiens britanniques (Moran, 2011; Baxter \& Jeffrey, 2013). On connait la place, aux États-Unis, des études post-mortem dans le développement des études sur le renseignement et notamment dans le parcours de chercheurs parmi les plus intéressants et les plus novateurs sur le sujet comme Richard Betts et Robert Jervis. En 2001, le directeur du Security Services décide de nommer un historien pour rédiger l'histoire officielle du service et de son action, en vue d'une publication pour le centenaire du MI 5. La mission est confiée à Christopher Andrew qui a pu s'appuyer sur un accès illimité aux documents ainsi que sur la possibilité de réaliser un très grand nombre d'entretiens avec les acteurs pour la réalisation de cette somme (Andrew, 2009). En 2006, le Secret Intelligence Service décida lui aussi de se lancer dans «l'aventure» d'une histoire officielle. La rédaction de l'ouvrage devant célébrer le centenaire du service est confiée à Keith Jeffery de la Queen's University, à Belfast. L'ouvrage est paru à la rentrée 2010 et ne couvre que la période 19091949 (Jeffery, 2010). Une histoire officielle du Joint Intelligence Commitee paraitra en 2013 sous la plume de Michael Goodman (Goodman, sous presse). Des histoires officielles du GCHQ et même du Defence Intelligence Staff, le moins connu des services secrets britanniques, sont en préparation.

Ces deux innovations majeures, si elles témoignent de l'ouverture croissante du monde du renseignement vers la recherche au RoyaumeUni, s'inscrivent dans une pratique britannique bien ancrée de composer l'histoire officielle des principaux conflits. Chaque guerre donne lieu à une histoire officielle, qui demeure parfois en tout ou en partie classifiée ou non diffusée, même si la dimension du renseignement est, sinon occultée, du moins négligée, comme dans le cas de la guerre des Malouines (Hinsley et al., 1979; Howard, 1990; Freedman, 2005).

\section{Grands axes de la recherche britannique}

L'objet de cette section est de présenter brièvement les grands axes de recherche développés par les sciences sociales sur les questions de renseignement au Royaume-Uni. Le corpus des études sur le renseignement au Royaume-Uni s'articule essentiellement autour de quatre thématiques structurantes. 
Comprendre la machine gouvernementale du renseignement et les institutions

Une part importante des travaux est consacrée à l'examen du fonctionnement de la machine gouvernementale en matière de renseignement et à une étude des différentes institutions qui participent à la politique de renseignement. Certains opus abordent le problème général de la politique du renseignement, de la place du renseignement et du secret au sein de l'État britannique (Thurlow, 1994; Hermann, 1996; Robertson, 1999; Hermann, 2001; Aldrich, 2009). Les deux services emblématiques ont fait l'objet d'études approfondies par des chercheurs, ainsi l'histoire officielle du MI 5 par Christopher Andrew (2009). Le MI 5 a, par ailleurs, fait l'objet d'une histoire non officielle très sérieuse et très bien documentée parue en 2009 (Hennessey \& Claire, 2009) Dans sa thèse consacrée à l'adaptation des services de renseignement au terrorisme islamiste, Frank Foley s'est focalisé sur le MI 5 en le situant dans une perspective plus large incluant le système judiciaire et les services de police et en recourant aux approches institutionnalistes, à la théorie des organisations internationales et au réalisme (Foley, 2009a, 2009b, 2013). Le MI 6 a été étudié par Philippe Davies (2004b) via la sociologie des organisations et l'analyse des politiques publiques. Les problèmes et les instances de coordination font l'objet d'une investigation croissante, à partir de l'ouvrage de référence de Peter Hennessy sur le fonctionnement de la mécanique gouvernementale face à la guerre froide (Hennessy, 2000; Field, 2009). Peter Gill a consacré plusieurs publications à l'Intelligence $\mathfrak{O}$ Security Committee (Glees et al., 2006; Gill, 2009b). On peut relever que le Defence Intelligence Staff et le GCHQ ne faisaient l'objet d'aucun travail de recherche de référence jusqu'à la publication récente de la monographie de Richard Aldrich (2010).

Par rapport aux Intelligence Studies américaines, l'approche britannique se distingue sur plusieurs points. Tout d'abord, la problématique de la politisation est beaucoup moins présente. Aux États-Unis, elle constitue quasiment un champ de recherche propre. Dans cette optique, l'étape de l'analyse est moins étudiée dans les travaux britanniques qu'américains où l'analyse et l'analyste sont toujours au cœur de la réflexion. De

6. Voir la note de lecture des deux ouvrages par Sergeant, J.-C. (2010). «Le MI 5 en 1684 pages. Le service de renseignement qui n'existait pas». Le Monde diplomatique, mars 2010 , p. 8. 
la même façon, la problématique de l'adaptation du système de renseignement et des services, l'enjeu de la réforme, est beaucoup moins obsidionale outre-Manche qu'outre-Atlantique. Plus fondamentalement, les études britanniques s'attachent à replacer la question du renseignement et les services de renseignement dans une analyse plus large de l'État et de ses transformations contemporaines et dans une économie générale de ses pratiques régaliennes. Sur ce point, les travaux de Davies sur le MI 6 (2004b) ou de Peter Hennessy (2000) sont les plus représentatifs. Le titre de certains travaux de références, The Secret State (Hennessy, 2010), The Vigilant State ${ }^{7}$, The Protective State (Hennessy, 2007), The Security State (Aldrich, 2009), Securing the State (Omand, 2010), témoignent de cette vigueur de la réflexion sur l'État et de la place qu'y tient le renseignement.

Interrogation démocratique et contrôle des services de renseignement

Le contrôle des services de renseignement, leur obligation de rendre des comptes (accountability), les modalités de l'Oversight, le contrôle parlementaire ou démocratique sur les institutions, l'action et les pratiques de l'État secret font l'objet d'une attention particulière au Royaume-Uni. Ce phénomène se renforce à travers les enquêtes récentes que nous avons détaillées précédemment. Certains ouvrages abordent une problématique large sur les relations entre renseignement, services secrets et démocratie (Gill, 1994; Leigh \& Lustgarten, 1994; Vincent, 1998; Robertson, 1999; Moran, 2013). D’autres études sont plus spécifiquement focalisées sur la question du contrôle parlementaire et de sa nature particulière dans le cas du renseignement puisque le Parlement est dépossédé de sa fonction d'Oversight au profit de l'Intelligence $\mathfrak{V}$ Security Committee (Robertson, 1988; Phythian, 2005b; Glees \& Davies, 2006; Gill, 2007a, 2007b; Phythian, 2007; Omand, 2008).

Récemment, la réflexion sur l'éthique de l'espionnage et du renseignement a fait l'objet d'un vrai renouveau intellectuel de la part de certains piliers de la communauté des études sur le renseignement au Royaume-Uni. Le contexte de l'usage de la torture dans la «guerre à la terreur», les dilemmes de la coopération avec des services étrangers pratiquant la torture y sont évidemment pour beaucoup. On peut aussi

7. Titre du module fondamental dispensé par Richard Aldrich à l'Université de Warwick. 
relever que ce sont principalement d'anciens praticiens, hauts fonctionnaires du renseignement, qui animent le débat: Michael Herman, David Omand, Michael Quinlan. Ils avancent notamment l'idée de forger une «théorie du juste espionnage» inspirée de la théorie de la guerre juste (Herman, 2004; Scott \& Jackson, 2004; Omand, 2006; Quinlan, 2007).

\section{La coopération internationale}

Certaines études ou témoignages offrent une perspective générale sur la coopération internationale du Royaume-Uni en matière de renseignement, y compris le renseignement électronique (Lander, 2004; Rudner, 2004; Walsh, 2007). Le cas américain à travers la problématique de l'Intelligence special relationship avec les États-Unis a principalement fait l'objet de travaux approfondis (Aldrich, 1998, 2001, 2002, 2004; Rees, 2006). La question de l'espionnage des alliés a notamment été le sujet d'un numéro spécial «Knowing Your Friends», Special issue of Intelligence and National Security, (vol. 13, $\left.\mathrm{n}^{\circ} 1,1998\right)$ sous la direction de Martin Alexander. On peut aussi noter un intérêt croissant pour la question du renseignement au sein de l'Union européenne (MüllerWille, 2002, 2008; Walsh, 2006).

Les problèmes soulevés par la coopération internationale en termes d'obligation de rendre des comptes et de contrôle parlementaire font l'objet d'une attention grandissante, notamment en raison de la problématique des extraditions forcées (extraordinary rendition) et de la torture dans la guerre à la terreur. Le jugement du cas de Binyam Mohamed, mettant en cause la coopération active d'agents des services britanniques avec des services étrangers pratiquant la torture dans le cadre et pour la politique américaine de guerre à la terreur, place cette question au cœur de l'agenda (Leigh, 2009; Deel, 2010).

Les travaux en cours de Robert Dover sur le rôle des services de renseignement dans la politique d'exportation d'armement du RoyaumeUni s'inscrivent aussi dans ce courant de recherche et devraient permettre de combler une lacune importante de la littérature sur la politique extérieure britannique (Dover, 2006, 2007).

\section{Les questions méthodologiques et théoriques}

Le diagnostic concernant la sous-théorisation des études sur le renseignement a été mis en lumière et discuté par Christopher Andrew 
(2004). Ce problème est au cœur de l'ouvrage de Peter Gill et Mark Phythian, Intelligence in an Insecure World (Gill \& Phythian, 2007). Le débat porte notamment sur la définition du renseignement par rapport à la notion de surveillance. Les travaux de science politique ont, en effet, tendance à replacer la question du renseignement et de ses services dans la problématique plus large de la surveillance, inspirée par les travaux de sociologues comme Giddens (1990) ou Dandeker (1990). Cette approche est notamment défendue par les auteurs «critiques» inspirés par les travaux de Foucault promouvant une conception postmoderne du renseignement (Rathmell, 2002; Gill \& Phythian, 2007), ou ceux liés au «Civil Liberties Project» (Dorril, 2000; Moran, 2011). Ce débat témoigne surtout du souci des chercheurs spécialistes du renseignement au Royaume-Uni de renforcer la dimension comme la portée théorique de leurs travaux. Un ouvrage important qui fait un tour d'horizon des principaux débats a été publié en 2008 sous la direction de chercheurs britanniques (Gill et al., 2008).

En termes méthodologiques, les chercheurs britanniques soulignent l'importance de mener des travaux comparatifs. Peter Gill a consacré un chapitre du récent Handbook of Intelligence Studies à la démarche comparative. Il conclut: «We really have no choice: if "intelligence studies" is to build on its growing store of historical accounts and institutional-legal descriptions, we need to get serious about theoretically informed comparative work both within and between nations and sectors» (Gill, 2009a : 88-89). Franck Foley (2009a) a ainsi récemment comparé les logiques d'adaptation des services français et britanniques face au terrorisme islamique. Phil Davies (2004a) compare les cultures nationales du renseignement britannique et américaine et leurs tendances à la collégialité ou au conflit à partir du cas irakien. Wyn Rees et Richard Aldrich (2005) ont comparé les cultures européenne et américaine de lutte contre le terrorisme.

\section{Comment expliquer le décalage de la productivité universitaire entre Royaume-Uni et France?}

Le constat est donc sans appel. En dépit d'importants développements au cours des 15 dernières années, les études sur le renseignement demeurent en France considérablement moins denses, diversifiées, nombreuses qu'au Royaume-Uni, alors qu'on peut estimer qu'il s'agit de deux pays très similaires en termes de qualité et d'importance de leurs 
services de renseignement. Comment expliquer ce triple décalage quantitatif, qualitatif et temporel? On se borne ici à avancer trois hypothèses qui n'ont pas été documentées à notre connaissance.

\section{Le fonctionnement du marché universitaire}

La première raison tient au fonctionnement du marché universitaire. Le renseignement, les services secrets demeurent un objet jugé peu légitime et peu pertinent.

Sans doute, pour une large part, le diagnostic posé par l'historien Alain Dewerpe, dans l'un des tout premiers ouvrages universitaires français à proposer une réflexion systématique dans ce domaine, demeure valable. Selon lui, il convient de «noter à quel point est grande la réticence du monde savant à s'engager dans un tel registre. Seule, peut-être, la politologie américaine, qui inscrit l'étude du "renseignement" dans une perspective étroitement fonctionnaliste, échappe à cette indifférence» (Dewerpe, 1994: 17). Au Royaume-Uni ou au Canada, les études sur l'intelligence se situent au carrefour de plusieurs autres disciplines, ou «champs universitaires » : War studies, Security studies (qui n'existent pas non plus en France), histoire et science politique. En ce sens, le renseignement est en France le dernier maillon d'une chaine de désintérêt ou d'intérêt relatif pour tout ce qui touche à la sphère régalienne: défense, sécurité, politique fiscale, politique étrangère. Tous ces sujets sont sous-étudiés en France par rapport aux autres grandes démocraties (États-Unis, Canada, Allemagne, Royaume-Uni). Au contraire, au Royaume-Uni, l'étude du renseignement bénéficie de la légitimité et à certains égards du prestige dont bénéficient les War Studies, l'histoire militaire, les relations internationales. De façon préoccupante, il n'y a pas en France de consensus sur le constat que le renseignement, ses services, ses acteurs, ses politiques soient «le chaînon manquant» pour comprendre la politique internationale française, les ressorts du pouvoir exécutif, les frontières de la gouvernance démocratique ou les fondements de la société de surveillance. Le milieu universitaire français est très largement réfractaire à l'étude des services secrets et du renseignement, encore considérée comme un objet peu légitime.

En France, les choses ont progressé en histoire, grâce à l'action résolue et continue de quelques figures marquantes telles que Olivier Forcade, à Saint-Cyr puis à Paris IV, et Sébastien Laurent, à Paris et à Bordeaux. En revanche, la science politique, la sociologie demeurent 
quasiment muettes sur ces sujets et semblent déterminées à le rester. En effet, si des recherches doctorales abordent les questions de renseignement, l'activité des services, souvent à partir de la question de la lutte anti-terroriste et de ses reconfigurations, le recrutement des jeunes enseignants-chercheurs travaillant sur ces thématiques demeure excessivement rare. Ce qui les conduit soit à l'expatriation, soit à trouver des solutions, souvent précaires, dans des institutions périphériques, mais pas à l'université ni au CNRS. Le phénomène étant connu et bien identifié, l'expatriation commence de plus en de plus dès la thèse. Pour le dire brutalement, faire une thèse sur le renseignement est un excellent passeport pour l'échec d'une carrière universitaire en France. L'absence de recrutement universitaire est bien évidemment un frein décisif au développement d'enseignements et de recherches sur les sujets et thématiques liés au renseignement et à l'obtention de toute masse critique.

La situation est bien différente, on l'a vu, au Royaume-Uni. On peut sans peine affirmer que disposer d'une thèse sur le renseignement est bien moins handicapant outre-Manche pour la réalisation d'une carrière universitaire. L'importance beaucoup plus forte que tient la demande étudiante dans la détermination de l'offre de formation et surtout dans la logique de recrutement de spécialistes du sujet au Royaume-Uni joue un rôle central.

\section{Transformation de l'État et reconnaissance du renseignement}

Le développement des études sur le renseignement au Royaume-Uni pendant les décennies 1980-1990 accompagne l'officialisation de l'existence des services secrets britanniques. Ce n'est qu'en 1989 que le Security Service (plus connu sous le nom de MI 5) a été légalement reconnu par l'intermédiaire du Security Service Act. Jusqu'en 1992, le gouvernement britannique ne reconnaissait pas officiellement l'existence d'un service de renseignement extérieur en temps de paix. L'Intelligence Services Act ne fournit une base légale à l'existence du Secret Intelligence Service (le fameux MI 6) ainsi qu'au service de renseignement chargé $\mathrm{du}$ renseignement électronique, le Government Communications Headquarters (GCHQ), qu'en 1994.

Cette reconnaissance se trouve à la conjonction de deux dynamiques: une dynamique structurelle de transformation de l'État britannique et de ses administrations vers une plus grande publicité, transparence et obligation de rendre des comptes sous l'effet du New Public Management, 
qui conduit par ailleurs chacune des administrations à devoir justifier ses dépenses comme ses résultats auprès des évaluateurs et financeurs (gouvernement, Parlement) ${ }^{8}$; une dynamique plus particulièrement propre aux agences de sécurité, générée par les pressions de la Cour européenne des droits de l'homme (CEDH). Les jugements de la CEDH, qui touchent beaucoup plus fortement le Royaume-Uni en raison de l'importance de la jurisprudence dans un pays de common law, imposent que les activités clandestines de surveillance, notamment les écoutes téléphoniques, soient autorisées par la loi et non par de simples décrets de l'Exécutif. La légalisation des services britanniques commence ainsi par une loi régissant les interceptions de communications en $1985^{9}$.

En France, le mouvement de «normalisation» du renseignement au sein de l'appareil d'État est bien plus tardif (Hayez, 2010). Il intervient pour l'essentiel à partir de 2007, à l'occasion de la préparation du Livre blanc sur la défense et la sécurité nationale. Alors qu'elle était tout à fait secondaire dans le livre blanc de 1994 (Long et al., 1994) et ne visait alors qu'à entériner le nouveau dispositif récemment mis en place à Creil avec la création de la DRM, de l'EMIA et du COS après la première guerre du Golfe, la fonction «connaissance et anticipation» devient dans le livre blanc sur la défense et la sécurité nationale de 2008 (Mallet, 2008) non seulement centrale, mais de surcroît structurante de la défense. Les termes classiques de la pensée militaire que sont «la connaissance préalable» et «l'information stratégique» reçoivent enfin leurs lettres de noblesse sous le vocable de «renseignement». Avec la refonte du renseignement intérieur dans une DCRI regroupant les RG et la DST dans une seule direction et communalisant leurs moyens techniques, la création d'un poste de coordinateur national du renseignement, placé institutionnellement entre le premier ministre et le président de la République, enfin la création en octobre 2007 d'une délégation parlementaire au renseignement commune au Sénat et à l'Assemblée nationale, le «renseignement» prend une place plus importante au sein de la politique nationale. Il est en outre de plus en plus présent dans l'espace public, et devient un objet moins obscur.

8. On observe ainsi une renaissance des doctrines militaires britanniques pendant cette période comme instrument de communication, même si ce n'est pas la seule raison de ce renouveau doctrinal.

9. Sur le passage de l'Intelligence Services Act de 1994, voir Gill (2011 : 43-63). 
(Re) légitimation du renseignement versus culture française du renseignement

Le développement des études sur le renseignement est aussi lié à la défense et à la légitimation des services de renseignement. Certains moments clés de la croissance des Intelligence Studies britanniques, depuis la fin de la guerre froide, notamment depuis 2003, sont liés au souci de permettre une meilleure compréhension publique de la nature du renseignement, de ses contraintes, tensions, limites et possibilités. Il s'agit d'une démarche visant à l'éducation du public concerné au premier chef d'une part, c'est-à-dire de l'ensemble des acteurs même périphériques qui peuvent avoir un rôle à jouer dans la collecte et la dissémination du renseignement (Omand, 2010). Mais d'autre part, elle vise également le public intéressé et plus largement l'ensemble de l'opinion publique. Michael Hermann rappelle ainsi que le développement des études sur le renseignement au cours des années 1970 provient de la frustration des anciens des services secrets de la Seconde Guerre mondiale face à l'interdiction officielle de mentionner leurs succès, comme l'opération Ultra et le rôle de Bletchley Park dans l'exploitation du code nazi Enigma ${ }^{10}$. Des recherches ultérieures permettront d'approfondir les attentes sociales en termes de bienfaits publics à l'endroit des études sur le renseignement: on peut par exemple envisager que les études universitaires sur le renseignement soient l'un des vecteurs de sensibilisation de l'électorat ou de mise en conformité de la représentation publique des services de renseignement avec leurs enjeux de recrutement futurs.

Depuis 2003, les projets d'histoire officielle des différents services fleurissent. Cette ouverture des services vers la recherche universitaire, à travers un accès contrôlé à leurs archives, vise à leur assurer une publicité plus sérieuse que celle divulguée par le folklore d'œuvres de journalistes ou de séries télévisées. Les anciens des services, en particulier de la Seconde Guerre mondiale, puis les services eux-mêmes avec l'histoire officielle ont joué un rôle déterminant dans le développement des études sur le renseignement au Royaume-Uni. Le contraste avec la France sur l'attitude des services est ici saisissant. Cette synergie entre les services et la recherche universitaire est incarnée par les deux figures marquantes que sont Michael Herman, auteur d'un maître livre sur la théorie du renseignement, qui anime depuis plusieurs années l'Oxford

10. Entretien de M. Hermann avec les auteurs, Oxford, juin 2010. 
Intelligence Group du Nuffield College et qui a exercé pendant plus de 30 ans au GCHQ ainsi que comme secrétaire du Joint Intelligence Committee et Sir David Omand, qui fut notamment le premier coordinateur du renseignement britannique (UK Security and Intelligence Co-ordinator) en tant que Permanent Secretary au Cabinet Office entre 2002 et 2005, aujourd'hui professeur au Department of War Studies du King's College et auteur du magistral Securing the State en 2010. Tous deux ont joué un rôle majeur d'animateur de la communauté de recherche sur le renseignement et de passerelle entre les services et le monde universitaire.

L'importance de la (re)légitimation des services et activités de renseignement se lit aussi dans le rôle que joue la révélation des scandales dans le développement des études sur le renseignement. La «saison des enquêtes» (Scott \& Jackson, 2004: 150), issue de l'engagement britannique dans la guerre d'Irak, et l'enquête sur les attentats de Londres de 2005 ont contribué à relancer les études sur le renseignement en leur donnant une matière empirique inhabituelle pour la période très contemporaine. Le rapport Hutton a fait l'objet d'une étude détaillée (Glees \& Davies, 2004). Les travaux s'appuyant sur ces enquêtes mettent l'accent sur les relations entre les décideurs politiques et les services de renseignement, ce qui est relativement novateur pour les études britanniques sur le renseignement (Davies \& Persbo, 2004; Freedman, 2004; Doig \& Phythian, 2005; Phythian, 2005b). L'analyse comparée des quatre rapports officiels montre les zones d'ombre qu'ont laissées les enquêtes officielles: elles portent notamment sur l'absence de certains services de renseignement, en particulier le GCHQ et le Defence Intelligence Staff du ministère de la Défense, ainsi que sur l'usage du renseignement par les décideurs politiques, le premier ministre en tête (Aldrich, 2005 ; O'Halpin, 2005). Une comparaison avec le rapport parlementaire australien souligne d'ailleurs l'absence de réflexion et d'investigation sur la politisation, évacuée dans les enquêtes officielles britanniques comme dans les enquêtes américaines (Gill \& Phythian, 2007).

En France, les «grandes affaires» de renseignement comme l'affaire du Rainbow Warrior n'ont pas donné lieu à des enquêtes, ni parlementaires ni indépendantes, qui auraient pu offrir une déclassification importante de documents à la disposition des chercheurs. Les rares missions d'information parlementaire qui traitent de sujets liés au renseignement, telles que la mission sur le Rwanda ou Srebrenica, sont 
particulièrement discrètes sur les activités de renseignement et le rôle des services secrets. La seule littérature faisant nettement exception est sans doute celle des mémoires «d'anciens» ou des «patrons» des services (voir sur l'intérêt et la portée de cette littérature qui représente depuis 1945 plus de quarante ouvrages: Laurent, 2011b). Le critère pertinent pour établir la spécificité des études françaises sur le renseignement résiderait donc dans un apparent consensus sur le paradigme de la «culture du renseignement». Celui-ci est quasi universellement évoqué, tant par les chercheurs que par les acteurs et témoins du renseignement. Les chercheurs étrangers qui s'intéressent au cas français reprennent et corroborent cette perception de soi sur le plan national, cette façon de penser les problématiques du renseignement et de poser le diagnostic d'un déficit en termes d'études et de recherche à leur endroit (Porch, 1997). Il faut se référer à un paradigme «de la culture du renseignement» plutôt qu'à un paradigme «culturaliste du renseignement». En effet, la discussion nationale qui a accompagné les premières études du renseignement ne s'est en rien apparentée à la position épistémologique du culturalisme tel qu'il existe dans les sciences humaines en général et dans la science politique en particulier. Ce thème, cette double formule de culture du renseignement ou de renseignement à la française, est l'endroit d'un paradoxe. Il y a doublement une culture française du renseignement, et la façon dont le problème a été pensé à l'initiative de l'amiral Lacoste le prouve assez. Le premier élément de la culture française du renseignement est précisément d'être incapable de penser le problème du renseignement en France autrement que sur le mode de l'absence de cette culture.

Le second élément réside dans le contenu même de la revendication de cette culture: il faut obtenir plus de reconnaissance pour les problématiques du renseignement, qui est déprécié si ce n'est proprement ignoré dans le reste de l'État, le monde économique et la société. Or les communautés du renseignement qui se sont développées ailleurs ont certes créé une «culture», mais elles l'ont fait en aval d'une institutionnalisation reposant sur des fonctions. S'il y a là-bas des cultures nationales dites du renseignement, c'est en lien avec la notion de communauté $\mathrm{du}$ renseignement dans l'État ou le gouvernement (Intelligence Communities). Le risque de l'approche «culturelle» à la française c'est qu'elle rend difficile la sortie d'un mode de perception de la situation, qui soit autre que le mode individualisé et psychologique. En ce sens, c'est seulement si une communauté du renseignement dans l'État finit par 
exister, à travers l'institution d'une académie du renseignement par exemple, qu'elle pourrait faire éclore une réelle «communauté épistémique» organisée autour de l'étude du renseignement. Sinon, la France risquerait de ne jamais sortir de son isolement et de ses démarches de recherche individuelles, quand bien même seraient-elles de grande ampleur. De ce point de vue, il serait tout à fait possible d'interpréter le consensus culturel sur les besoins d'en sortir comme l'expression d'un besoin de reconnaissance d'un «corps» en puissance au sein de l'État français qui tarde à se penser et à se déclarer comme tel.

L'intérêt des travaux publiés le plus récemment consiste donc à proposer enfin un dépassement de ce paradoxe autour de la «culture» $\mathrm{du}$ renseignement. Ils dégagent petit à petit le renseignement de la gangue de ses problématiques culturelles pour les lier plus en profondeur à la catégorie d'État (voir sur ce point: Jackson, 2006; Chopin et al., 2011). En tentant de dégager les caractéristiques de cette singulière «école française» en redéploiement, on obtiendrait donc quatre vecteurs d'évolution significatifs: une exigence particulière de documentation et d'appui sur les archives; par conséquent, une articulation plus rigoureuse des études de cas adossées à une réflexion théorique; un recentrage, assez proche de celui des Britanniques, des pratiques du renseignement autour de la collecte (ou recueil) plutôt que de l'analyse; une volonté d'établir un lien entre le plan de l'État pris dans sa globalité et les pratiques politiques particulières étudiées avec la plus grande précision (un heureux entre-deux entre théorisation générale et études de cas).

\section{Références}

Aldrich, R. (1998). British Intelligence and the Anglo-American "Special Relationship" during the Cold War. Review of International Studies, 24 (3), 331-351.

Aldrich, R. (2001). Hidden Hand: Britain, America and the Cold War secret Intelligence. Londres: John Murray.

Aldrich, R. (2002). Dangerous Liaisons: Post-September 11 Intelligence Alliances. Harvard International Review, 24 (3), 50-54.

Aldrich, R. (2004). Transatlantic Intelligence and security cooperation. International Affairs, 80 (4), 731-754.

Aldrich, R. (2005). Whitehall and the Iraq War: The UK's four Intelligence enquiries. In C. Andrew, R. Aldrich \& W. Wark (eds.), Secret Intelligence. A Reader. Londres: Routledge. 
Aldrich, R. (2009). The security state. In M. Flinders, A. Gamble, C. Hay \& M. Kenny (eds.), The Oxford Handbook of British Politics. Oxford: Oxford University Press.

Aldrich, R. (2010). GCHQ. The uncensored story of Britain's most secret Intelligence agency. New York \& Londres: Harper \& Collins.

Andrew, C. (2004). Intelligence, international relations and "Under-Theorisation". In L. Scott \& P. Jackson (eds.), Understanding Intelligence in the TwentyFirst Century: Journeys in the Shadow (170-184). Londres: Routledge.

Andrew, C. (2009). In defence of the realm. The authorized history of MI5. Londres: Penguin.

Arboit, G. (2013). Le renseignement, dimension manquante de l'bistoire contemporaine de la France (Rapport $\left.n^{\circ} 9\right)$. Paris: CF2R.

Baxter, C., \& Jeffery, K. (2013). Intelligence and official history. In C. Moran $\&$ C. Murphy (eds.), Intelligence Studies in Britain and the United States. Historiography since 1945 (289-303). Edinburgh: Edinburgh University Press.

Bonditti, P. (2007). L'anti-terrorisme aux États-Unis (1947-2007). Une analyse foucaldienne de la transformation de l'exercice de la souveraineté et de l'art de gouverner (Thèse de doctorat). Sciences Po, Paris.

Chopin, O. (2005). La raison d'État et la démocratie, concepts et pratiques (Thèse de doctorat). L'École des hautes études en sciences sociales, Paris.

Chopin, O., Irondelle, B., \& Malissard, A. (2011). Étudier le renseignement. État de l'art et perspectives de recherche (Étude $\mathrm{n}^{\circ}$ 9). Paris: IRSEM.

Dandeker, C. (1990). Surveillance, power and modernity. New York: St. Martin's Press.

Davies, P. (2004a). Intelligence culture and intelligence failure in Britain and the United States. Cambridge Review of International Affairs, 17 (3), 495-520.

Davies, P. (2004b). MI 6 and the Machinery of Spying. Londres: Franck Cass.

Davis, I., \& Persbo, A. (2004). After the Butler report: time to take on the Group Think in Washington and London. BASIC papers: occasional papers in international security, 46.

Deel, S. (2010). Intelligence cooperation and torture. International Law Discussion Group Meeting Summary. Londres: Chatam House.

Dénécé, E., \& Arboit, G. (2010). Intelligence Studies in France. International Journal of Intelligence and Counter-Intelligence, 23 (4), 725-747.

Dewerpe, A. (1994). Espion. Une anthropologie historique du secret d'État contemporain. Paris: Gallimard.

Doig, A., \& Phythian, M. (2005). The national interest and the politics of threat exaggeration: The Blair government's case for war against Iraq. Political Quarterly, 76 (1), 368-376.

Dorril, S. (2000). MI6: Fifty Years of Special Operations. Londres: Fourth Estate.

Dover, R. (2006). A silent debate: The role of Intelligence in the UK Arms Trade. International Journal of Intelligence and CounterIntelligence, 19 (1), 110119.

Dover, R. (2007). For Queen and Company - The Role of Intelligence in the UK's Arms Trade. Political Studies, 55 (4), 683-708. 
Faligot, R., Guisnel J., \& Kauffer, R. (2012). Histoire politique des services secrets français. Paris: La Découverte.

Field, A. (2009). Tracking terrorist networks: Problems of intelligence sharing within the UK intelligence community. Review of International Studies, 35, 997-1009.

Foley, F. (2009a). Reforming conterterrorism: Institutions and organizational routines in Britain and France. Security Studies, 18 (3), 435-478.

Foley, F. (2009b). The expansion of intelligence agency mandates: British counter-terrorism in comparative perspective. Review of International Studies, 35, 983-995.

Foley, F. (2013). Countering terrorism in Britain and France. Cambridge: Cambridge University Press.

Forcade, O. (2001). L'exploitation du renseignement stratégique en 1936-1938. In G.H. Soutou, J. Frémeaux \& O. Forcade (eds.), L'exploitation du renseignement en Europe et aux États-Unis des années 1930 aux années 1960 (83-98). Paris: Économica.

Forcade, O. (2005). Michel Debré et les fins politiques du renseignement 1959-1962. Communication présentée au colloque Michel Debré, chef de gouvernement du CHEVS-FNSP, Palais du Luxembourg, Paris.

Forcade, O. (2008). La République secrète. Histoire des services spéciaux militaires français de 1918 à 1939. Paris: nouveau monde éditions.

Forcade, O., \& Laurent, S. (2005). Secrets d'État: Pouvoirs et renseignement dans le monde contemporain. Paris: Armand Colin.

Forcade, O., Rivet, L., \& Laurent, S. (2010). Carnets du chef des services secrets 1936-1944. Paris: nouveau monde éditions.

Freedman, L. (2004). War in Iraq: Selling the threat. Survival, 46 (2), 7-50.

Freedman, L. (2005). The official bistory of the Falklands Campaign. Londres: Stationary Office.

Giddens, A. (1990). Consequences of Modernity. Cambridge: Polity.

Gill, P. (1994). Policing politics: Security Intelligence and the Liberal Democratic State. Londres: Frank Cass.

Gill, P. (2007a). Democratic and parliamentary accountability of Intelligence Services after September $11^{\text {th }}$. Geneva Centre for Democratic Control of Armed Forces, Working Paper 103.

Gill, P. (2007b). Evaluating intelligence oversight committees: The UK Intelligence and Security Committee and the "war on terror". Intelligence and National Security, 22 (1), 14-37.

Gill, P. (2009a). Knowing the self, knowing the other: The comparative analysis of security intelligence. In L. Johnson (ed.), Handbook of Intelligence Studies (82-90). Londres: Routledge.

Gill, P. (2009b). The intelligence and security committee and the challenge of security networks. Review of International Studies, 35, 929-941.

Gill, P. (2011). A formidable power to cause trouble for the governement? Intelligence oversight and the creation of the UK Intelligence and Security 
Committee. In R. Dover \& M. Goodman (eds.), Learning from the Secret Past. Cases in British Intelligence History (43-63). Washington DC: Georgetown University Press.

Gill, P., Marrin, S., \& Phythian, M. (2008). Intelligence theory: Key questions and debates. Londres: Routledge.

Gill, P., \& Phythian, M. (2007). Intelligence in an Insecure World. Cambridge: Polity.

Glees, A., \& Davies, P. H. J. (2004). Spinning the spies. Intelligence, open government and the Hutton inquiry. Londres: Social Affairs Unit.

Glees, A., \& Davies, P. H. J. (2006). Intelligence, Iraq and the limits of legislative accountability during political crisis. Intelligence and National Security, 21 (5), 848-883.

Glees, A., Davies, P., \& Morrison, J. (2006). The open side of secrecy: Britain's Intelligence and Security Committee. Londres: Social Affair Unit, 2006.

Goodman, M. (2006). Studying and teaching about intelligence: The approach in the UK. CIA Studies in Intelligence, 50 (2), 57-66.

Goodman, M. (sous presse). The official history of the joint Intelligence Committee. Vol. I From the approach of World War II to the Suez crisis. Londres: Routledge.

Goodman, M., \& Omand, D. (2008). Teaching intelligence analysts in the UK. What analysts need to understand: The King's intelligence studies program. CIA Studies in Intelligence, 52 (4), 1-12.

Guittet, E.-P. (2006). La genèse de la coopération anti-terroriste en Europe et l'implication de l'Espagne dans la (re)définition de l'identité européenne (Thèse de doctorat). Sciences Po, Paris.

Hayez, P. (2010). «Renseignement»: The new French intelligence Policy. International Journal of Intelligence and Counterintelligence, 23, 474-486.

Hennessy, P. (2000). The secret state: Whitehall and the Cold War, 1945-1970. Londres: Allen Lane.

Hennessy, P. (Ed.). (2007). The new protective state. Government, intelligence and terrorism. Londres: Continuum.

Hennessy, P. (2010). The secret state. Preparing for the worst, 1945-2010. Londres: Penguin Books.

Hennessey, T., \& Claire, T. (2009). The unofficial bistory of MI 5. Londres: Amberley Publishing.

Her Majesty's Stationery Office (HMSO). (2004). Review of intelligence on weapons of mass destruction, HC898. Londres: HMSO

Her Majesty's Stationery Office (HMSO). (2005). Review of intelligence on weapons of mass destruction: Implementation of its conclusions, CM 6492. Londres: HMSO.

Herman, M. (1996). Intelligence power in peace and war. Cambridge: Cambridge University Press.

Herman, M. (2001). Intelligence Services in the Information Age. Londres: Frank Cass.

Herman, M. (2004). Ethics and intelligence after September 2001. Intelligence and National Security, 19 (2), 342-358. 
Hinsley, H. F, Thomas, E. E., Simkins, C. A. G., \& Ransom, C. F. G. (1979). British Intelligence in the Second World War. Its Influence on Strategy and Operations. Londres: HMSO.

Howard, M. (1990). British intelligence in the Second World War. Londres : Stationary Office.

Jackson, P. (2006). Intelligence and the state: An emerging "French school" of Intelligence Studies. Intelligence and National Security, 21 (6), 1061-1065.

Jackson, P. (Ed.). (sous presse). Intelligence studies. A 5 volume-set. Londres: Sage.

Jeffery, K. (2010). MI 6. The history of the Secret Intelligence Service 1909-1949. Londres: Bloomsburry Publishing.

Johnson, L. (2006). Strategic intelligence. Greenwood: Praeger.

Johnson, L. (Ed.). (2010). The Oxford handbook of national security intelligence. Oxford: Oxford University Press.

Kahn, D. (2008). Intelligence studies on the continent. Intelligence and National Security, 23 (2), 249-275.

Lacoste, P. (Ed.). (1997). Approches françaises du renseignement: y a-t-il une «culture» nationale? Paris: Fondation pour les études de défense.

Lacoste, P. (Ed.). (1998). Le renseignement à la française. Paris: Économica.

Lander, S. (2004). International intelligence cooperation: An insider perspective. Cambridge Review of International Studies, 17 (3), 481-493.

Laurent, S. (Ed.). (2003). Archives "secrètes», secrets d'archives? L'bistorien et l'archiviste face aux archives sensibles. Paris: CNRS éditions.

Laurent, S. (Ed.). (2009a). Politiques du renseignement. Bordeaux: Presses universitaires de Bordeaux.

Laurent, S. (2009b). Entre l'État et le marché: l'information et l'intelligence économique en France. Paris: nouveau monde éditions.

Laurent, S. (2009c). Politiques de l'ombre. État, renseignement et surveillance en France. Paris: Fayard.

Laurent, S. (Ed.). (2011a). Politiques sous surveillance. Bordeaux: Presses universitaires de Bordeaux.

Laurent, S. (Ed.). (2011b). Les Espions français parlent. Archives et témoignages inédits des services secrets français. Paris: Nouveau monde éditions.

Leigh, I. (2009). Changing the rules of the game: Some necessary legal reforms to United Kingdom Intelligence. Review of International Studies, 35, 943-955.

Leigh, I., \& Lustgarten, L. (1994). In from the cold: National security and democracy. Oxford: Oxford University Press.

Lepri, C. (sous presse). Le contrôle parlementaire des services de renseignement: enjeux et perspectives (Thèse de doctorat). Paris 8 , France.

Long, M., Balladur, É., \& Leotard, F. (1994). Livre Blanc sur la Défense 1994. Paris: La documentation Française.

Mallet, J.-C. (2008). Défense et Sécurité nationale: le livre blanc. Paris: La documentation Française. 
Masterman, J. C. (1972). The double-cross system in the War 1939-1945. Londres: Yale University Press.

Moran, C. (2013). Classified. Secrecy and the state on modern Britain. Cambridge: Cambridge University Press.

Moran, M. (2011). The pursuit of intelligence history: Methods, sources, and trajectories in the United Kingdom. Studies in Intelligence, 55 (2), 33-55.

Müller-Wille, B. (2002). EU intelligence cooperation: A critical analysis. Contemporary Security Policy, 23 (2), 61-86.

Müller-Wille, B. (2008). The effect of international terrorism on EU intelligence co-operation. Journal of Common Market Studies, 46 (1), 49-73.

O'Halpin, E. (2005). British intelligence and the case for confronting Iraq: Evidence from the Butler and Hutton reports. Irish Studies in International Affairs, 16, 89-102.

Omand, D. (2006). Ethical guidelines in using secret intelligence for public security. Cambridge Review of International Affairs, 19 (4), 613-628.

Omand, D. (2008). Can we have the pleasure of the grin without seeing the cat? Must the effectiveness of secret agencies inevitably fade on exposure to the light? Intelligence and National Security, 23 (5), 593-607.

Omand, D. (2010). Securing the State. Londres: Hurst.

Phythian, M. (2005a). Still a matter of trust: Post-9/11 British intelligence and political culture. International Journal of Intelligence and CounterIntelligence, 18 (4), 653-681.

Phythian, M. (2005b). Hutton and Scott: A tale of two inquiries. Parliamentary Affairs, 58 (1), 124-137.

Phythian, M. (2007). The British experience with intelligence accountability. Intelligence and National Security, 22 (1), 75-99.

Porch, D. (1997). Histoire des services secrets français. 2 tomes. Paris: Albin Michel.

Quinlan, M. (2007). Just intelligence: Prolegomena to an ethical theory. In P. Hennessy (ed.), The New Protective State. Government, Intelligence and Terrorism (123-141). Londres: Continuum.

Rathmell, A. (2002). Towards postmodern intelligence. Intelligence and National Security, 17 (3), 87-104.

Rees, W. (2006). Transatlantic security cooperation: Drugs, crime and terrorism in the twenty-first century. Londres: Routldge.

Rees, W., \& Aldrich R. (2005). Contending cultures of counter-terrorism: Divergence or convergence? International Affairs, 81 (5), 905-924.

Robertson, K. G. (1988). Accountable intelligence: The British experience. Conflict Quarterly, 8 (1), 13-28.

Robertson, K. G. (1999). Secrecy and open government: Why governments want you to know. Londres: MacMillan.

Rudner, M. (2004). Britain betwixt and between: UK SIGINT alliance strategy's transatlantic and European connections. Intelligence and National Security, $19(4), 571-590$. 
Scott, L., \& Jackson, P. (2004). The study of intelligence in theory and practice. Intelligence and National Security, 19 (2), 139-169.

Thurlow, R. (1994). Secret state. British Internal Security in Twentieth Century. Oxford Blackwell.

Vadillo, F. (2012). L'Élysée et l'exercice du pouvoir sous la Ve République: le cas de la politique de lutte anti-terroriste (1974-1997) (Thèse de doctorat). Sciences Po Bordeaux, France.

Vincent, D. (1998). The culture of secrecy, Britain 1832-1998. Oxford: Oxford University Press.

Walsh, J. (2006). Intelligence-sharing in the European Union: Institutions are not enough. Journal of Common Market Studies, 44 (3), 625-643.

Walsh, J. (2007). Defection and hierarchy in international intelligence sharing. Journal of Public Policy, 27 (2), 151-181.

Warusfel, B. (Ed.). (1998). Il n'est point de secret que le temps ne révèle: études sur l'bistoire du renseignement. Paris: Lavauzelle.

Warusfel, B. (Ed.). (2007). Le renseignement: guerre, technique et politique (XIX ${ }^{e}-$ $X X^{e}$ siècles). Paris: Lavauzelle.

Winterbotham, F. C. (1974). The ultra secret. Londres: Weindenfeld and Nicolson.

ABSTRACT - As the increasing number of handbooks on intelligence studies indicates, internationally political science has reached a certain level of maturity. However, in France, academic research in this field is in its infancy. The French academic research not only started to develop long after intelligence studies in Britain, but France is also unique because of a paradox: While public knowledge is weak, France is one of the few countries to run a full range of intelligence activities and organisations, both civil and military, human and technical, etc. This paper presents the current state of research on intelligence in France and the United Kingdom, analyzing the developments in this field in both countries. It speculates on the British achievements compared to the late and fragile development of French intelligence studies. These differences can be explained by many factors including the functioning of the academic market, the transformation of the state and recognition of intelligence, and, finally, the legitimization of intelligence on both sides of the Channel.

KEYWORDS - Intelligence, intelligence studies, intelligence agencies, France, United Kingdom.

RESUMEN - Mientras que la publicación, devenida "industrial", de manuales sobre los Estudios de Inteligencia demuestra la maduración de los estudios sobre las tareas de inteligencia en el seno de la ciencia política internacional, la investigación académica sobre el tema permanece, en Francia, en estado emergente. Al atraso francés en relación al Reino Unido se agrega un segundo desfase entre el poco conocimiento e importancia de los servicios de inteligencia, siendo Francia uno de los pocos países en disponer de una panoplia completa de servicios de inteligencia interiores y exteriores, civiles y militares, y de recursos técnicos líderes. El artículo presenta el estado actual de los estudios sobre inteligencia en Francia y en el Reino Unido, analiza la trayectoria 
del desarrollo de dicho campo de investigación y adelanta hipótesis sobre la exitosa historia británica y el tardío y frágil desarrollo de los estudios sobre inteligencia en Francia. La explicación radica en el funcionamiento del mercado académico, en las transformaciones del Estado, en las modalidades del reconocimiento institucional y social de la inteligencia, y finalmente, en los mecanismos de un proceso diferenciado de relegitimación de la inteligencia sobre las dos riberas de la Mancha.

PALABRAS CLAVE - Inteligencia, estudios sobre la inteligencia, servicios secretos, Francia, Gran Bretaña. 